\title{
Impact of the Large-Scale Solar Magnetic Field on the Solar Corona and Solar Wind
}

\author{
A.G. Tlatov ${ }^{1}$ and B.P. Filippov ${ }^{2}$ \\ ${ }^{1}$ Kislovodsk Mountain Station of the Central \\ Astronomical Observatory of RAS at Pulkovo \\ ${ }^{2}$ Pushkov Institute of Terrestrial Magnetism, \\ Ionosphere and Radio Wave Propagation, \\ Russian Academy of Sciences, Troitsk, Moscow Region \\ Russia
}

\section{Introduction}

In 1955 Soviet astrophysicists Vsehsvyatskiy, Nikolskiy, Ponomarev and Cherednichenko (Vsekhsvyatskiy et al., 1955) showed that broad corona loses its energy for radiance, and can be in hydrodynamic equilibrium, and there should be a flow of materials and energy.

This process is a physical basis for the important phenomenon of "dynamic corona". The magnitude of the flow of materials was evaluated due to the following considerations: if the corona were in hydrodynamic equilibrium, then the altitudes of homogenous atmosphere for hydrogen and iron would correlate as 56/1. In other words, in such case iron ions must not be observed in the distant corona. But this is not so. In 1955 it was a considerable achievement, but nobody believed in the phenomenon of "dynamic corona".

Three years later Eugene N. Parker came to the conclusion that hot solar stream in Chapman model and particle flux, blowing away commentary tails in Birmann's hypothesis - these are manifestation of the same phenomenon, and Eugene N. Parker called it "solar wind".

Parker (Parker, 1958) showed - despite the fact that solar corona is greatly gravitated to the Sun, it is a strong heat conductor, it remains hot even at great distance. The farther the distance, the less the solar gravitation is, there is a supersonic discharge from the upper corona into interplanetary space.

Solar wind represents a flux of ionized particles, thrown out of the Sun in all the directions with the speed about $300-1200 \mathrm{~km} / \mathrm{sec}$. The source of the solar wind is solar corona. The temperature of the solar corona is so high, that gravitation force is not able to hold its substance near the surface, and part of this substance constantly moves to interplanetary space.

First direct gaging of the solar wind was carried out in 1959 by the automatic interplanetary station "Luna-1". The observations were made by means of a scintillometer and a gas ionization detector. Three years later the same gaging was implemented by the American scientists on board the station "Mariner-2". 
First numerical models of solar wind in corona with using the equations of magnetofluid dynamics were created by Pneuman and Kopp in 1971 (Pneuman \& Kopp, 1971).

In the end of the 90s there were observations of the areas of uprise of fast solar wind in solar poles, those observations were made on board of the satellite $\mathrm{SOHO}$ by means of Ultraviolet Coronal Spectrometer (UVCS). It turned out that acceleration of the wind is much higher than it was presupposed, judging from purely thermodynamic extension. Parker's model predicted that the wind speed becomes hypersonic in 4 solar radii altitude from photosphere, but the observations showed that this transition takes place significantly lower, approximately in 1 solar radius altitude, confirming the existence of the extra mechanism of acceleration of the solar wind.

Long-term observations from the orbit of the Earth (about $150000000 \mathrm{~km}$ distance from the Sun) showed that the solar wind is structured and can usually be classified as steady and perturbative (sporadic and recurrent).

Depending on the speed, sporadic streams of solar wind can be divided into slow (approximately $300-500 \mathrm{~km} / \mathrm{sec}$ near the orbit of the Earth) and fast $(500-800 \mathrm{~km} / \mathrm{sec}$ near the orbit of the Earth) (Fig. 1).

Slow solar wind is generated by the "steady" part of the solar corona (area of coronal streamers) with its gas-dynamic extension: with the temperature of corona about $2 \times 10^{6} \mathrm{~K}-$ cjrona cannot be in the condition of hydrostatic equilibrium, and this extension under the present border conditions must lead to the acceleration of the corona substance up to hypersonic speeds.

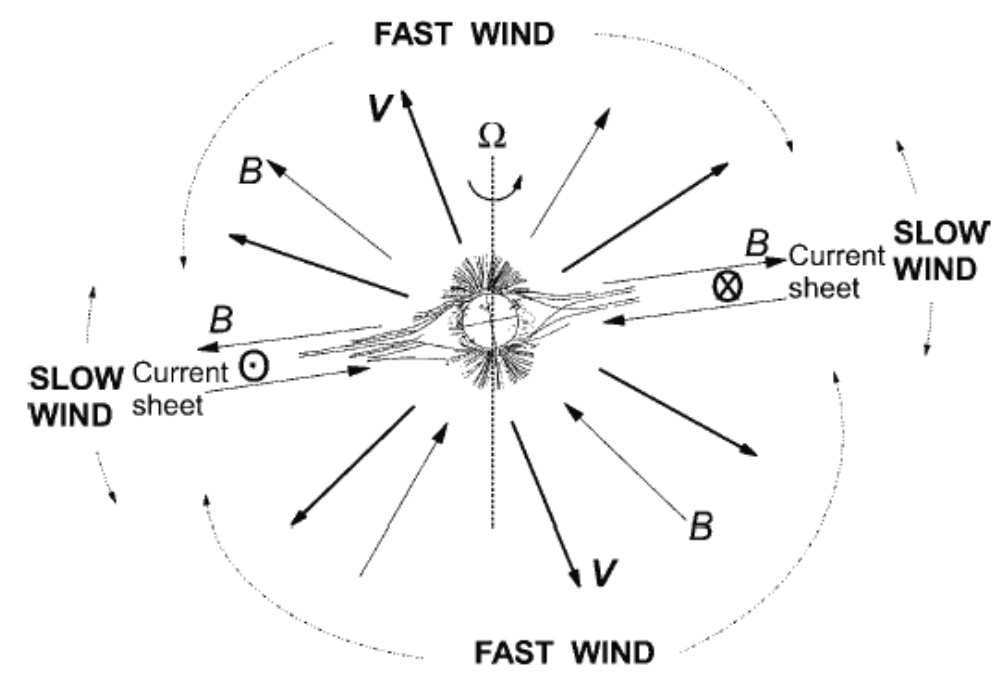

Fig. 1. Simplified picture of the large-scale structure of the solar wind near sunspot minimum, when the solar magnetic dipole makes a small angle with the spin axis (dotted line). The velocity and field lines are sketched in bold and thin lines respectively. The magnetic polarity is the one that existed when the WIND and Ulysses observations were acquired; this polarity reverses every 11 years (Meyer-Vernet, 2007). 
Solar corona heating up to such temperatures takes place due to convectional nature of heat transfer in the solar photosphere: the development of the convective turbulence in plasma is accompanied by the generation of intensive magnetoacoustic waves. In its turn, during transmission towards solar atmosphere density reduction, sound waves are transforming into shock waves; shock waves are effectively absorbed by the corona substance and heat it till the temperature of $(1-3) \times 10^{6} \mathrm{~K}$. The streams of recurrent fast solar wind are emanated by the Sun during several months and have a return period of 27 days (the period of the rotation of the Sun) when being observed from the Earth. These streams are associated with coronal holes - the areas of the corona with relatively low temperature (approximately $0,8 \times 10^{6} \mathrm{~K}$ ), low-density of plasma (that is all in all a quarter of density of the sporadic corona areas) and radial in relation to the Sun magnetic field. Owing to high conductivity of the solar wind plasma magnetic field of the Sun turns out to be frozen-in into the flowing out wind streams, and can be observed within the interplanetary atmosphere as an interplanetary magnetic field.

Because of the solar wind the Sun loses about one million tons of its substance every second. The solar wind consists basically of electrons, protons hellions (alpha-particles); nuclei of other elements and non-ionized particles (electrically neutral) are contained in rather inconspicuous quantity.

\section{Long-term changes of coronal shape and geomagnetic disturbance}

Solar wind parameters are changing during the solar activity. Their direct determination has begun relatively recently, with the beginning of space age. But the observations of the geomagnetic disturbance and coronal shape give us the opportunity to evaluate variations of the solar wind throughout the period of more than 100 years.

The large-scale solar corona structure corresponds to the large-scale configuration of solar magnetic fields. Since the magnetic field of the Sun is subjected to cyclic variations, the coronal shape also changes cyclically. Processing 12 photographs of the corona during solar eclipses, Ganskiy (Ganskiy, 1897) classified 3 types of corona, i.e., maximum, intermediate, and minimum. In 1902, in the report concerning the solar eclipse of 1898, Naegamvala (Naegamvala, 1902) also gave a corona classification that depended on the sunspot activity. Description of the corona shape involves the use of characteristic features and the phase of solar activity that is given by $\Phi=\frac{T-T_{\min }}{\left|T_{\max }-T_{\min }\right|}$. The values of $\Phi$ are positive and negative at the rising and declining branches of the solar cycle. Vsekhsvyatskiy (Vsekhsvyatskiy et al., 1965) gave a somewhat different classification of the structure types. They are (1) a maximum type $\Phi>0.85$ in which polar-ray structures are not seen, large streamers are observed at all heliolatitudes and are situated radially; (2) an intermediate premaximum or postmaximum type $0.85>|\Phi|>0.5$, in which polar-ray structures are observed at least in one hemisphere, and large coronal streamers situated almost radially are clearly seen at high latitudes; (3) an intermediate preminimum or postminimum type $0.5>|\Phi|>0.15$ in which polar-ray structures are clearly seen in both hemispheres and large coronal streamers strongly deviate toward the solar equator plane; (4) a minimum type $0.15>|\Phi|$ in which polar-ray structures are clearly seen in both hemispheres and large coronal streamers are parallel to the equator plane; and (5) an ideally minimum type $0.05>|\Phi|$ in which 
powerful structures of large coronal streamers are situated along the equator. Changes in the extent of the polar-ray structures, the degree of corona flattening, the average angle between large coronal streamers, and other characteristics of the corona that depend on the solar cycle phase have been widely studied (Loucif and Koutchmy, 1989, Vsekhsvyatskiy et al., 1965, Golub \& Pasachoff, 2009).

Solar eclipses of 2006 Mar 26, 2008 Jul 22, and 2009 Aug 1 have enabled detailed examination of changes in the shape of the corona in the period of minimum solar activity in the modern era. However, the shape of the corona in the current minimum is slightly different from the ideal shape of the eclipses in the minimum of activity.

This section contains the comparative analysis of the solar corona structure during the minimums of activity cycles 12-24, and their changes throughout the centuries are discussed.

\subsection{Processing method and results}

The initial data in the analysis were drawings of the corona shape taken from the catalogs of (Vsekhsvyatskiy et al., 1965) (see Fig. 3, 1-8), Loucif and Koutchmy, 1989) (Fig. 3, 9) and also drawings of the eclipses at minima of cycles 21 and 23 taken from Waldmeier (Waldmeier, 1976) ( Fig. 3, 10), Gulyaev (Gulyaev, 1997) (Fig. 2, 11) (Eclipse photo of 01.08.2008 with permission of M. Druckmüller), (Fig. 2,12). (Vsekhsvyatskiy et al., 1965) separated out an ideally minimum corona. It is supposed that the cycle phase must be $|\Phi|<0.05$. In fact, the ideally minimum corona type was observed on in 1954 (see Fig. 3). This happened at the solar activity minimum before the largest one in the history of observation cycle 19. Most probably, such a corona type did not occur for 50 years before and after this event. The corona shape of 1954 was close to a dipole one. This means that large coronal streamers rapidly approach the solar equator plane. However, during other eclipses, such as 1889 Jan 1 $(\Phi=-0.18), 1889 \operatorname{Dec} 21(\Phi=0.03), 1901$ May $17(\Phi=-0.07), 1923$ Sep $10(\Phi=0.04)$, and 1965 May $30(\Phi=0.14)$, the large coronal streamers at distances more than 2 solar radii do not lie on the equator plane. They expand from mid-latitudes in parallel or at a slight angle to the equatorial plane (see Fig. 3). To analyze the corona shape at the eclipses during a solar activity minimum epoch, a corresponding index should be chosen. We need the index that characterizes the shape of the corona of the minimal type and is applicable to images and drawings of different qualities. The corona of the solar minimum is characterized by pronounced polar ray structures and large coronal streamers (Pasachoff et al., 2008). Let us introduce the parameter $\gamma$ that characterizes the angle between high-latitude boundaries of the large coronal streamers at a distance of $2 \cdot R_{\odot}$. The $\gamma$ parameter is the sum of the angles at the eastern and western limbs: $\gamma=180-\left(\gamma_{W}+\gamma_{E}\right)$. Figure 2 gives the scheme showing how parameter $\gamma$ is determined from the shapes of the corona in Fig. 3, and the error does not exceed $4^{\circ}$.

The parameter $\gamma$ is close to the index of the extent of the polar regions (Nikolskiy 1955; Loucif \& Koutchmy, 1989; Golub, 2009). But in comparison with the polar index (Loucif \& Koutchmy, 1989), it is not calculated close to the limb, but at a height $R_{\odot}$ above the solar limb. This allows taking the compression of coronal rays to the plane of which is the equator into account, possibly linked with non-radial spreading of the coronal streamers. This is especially significant for the minimum activity epoch (Tlatov, 2010a). 


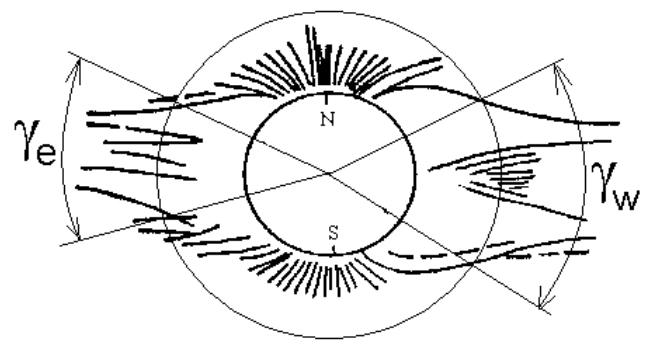

Fig. 2. Scheme showing how the angles defining parameter $\gamma=180-\left(\gamma_{W}+\gamma_{E}\right)$ are found for the eclipse of 1923. The outer thin circle has a radius of $2 R_{o}$

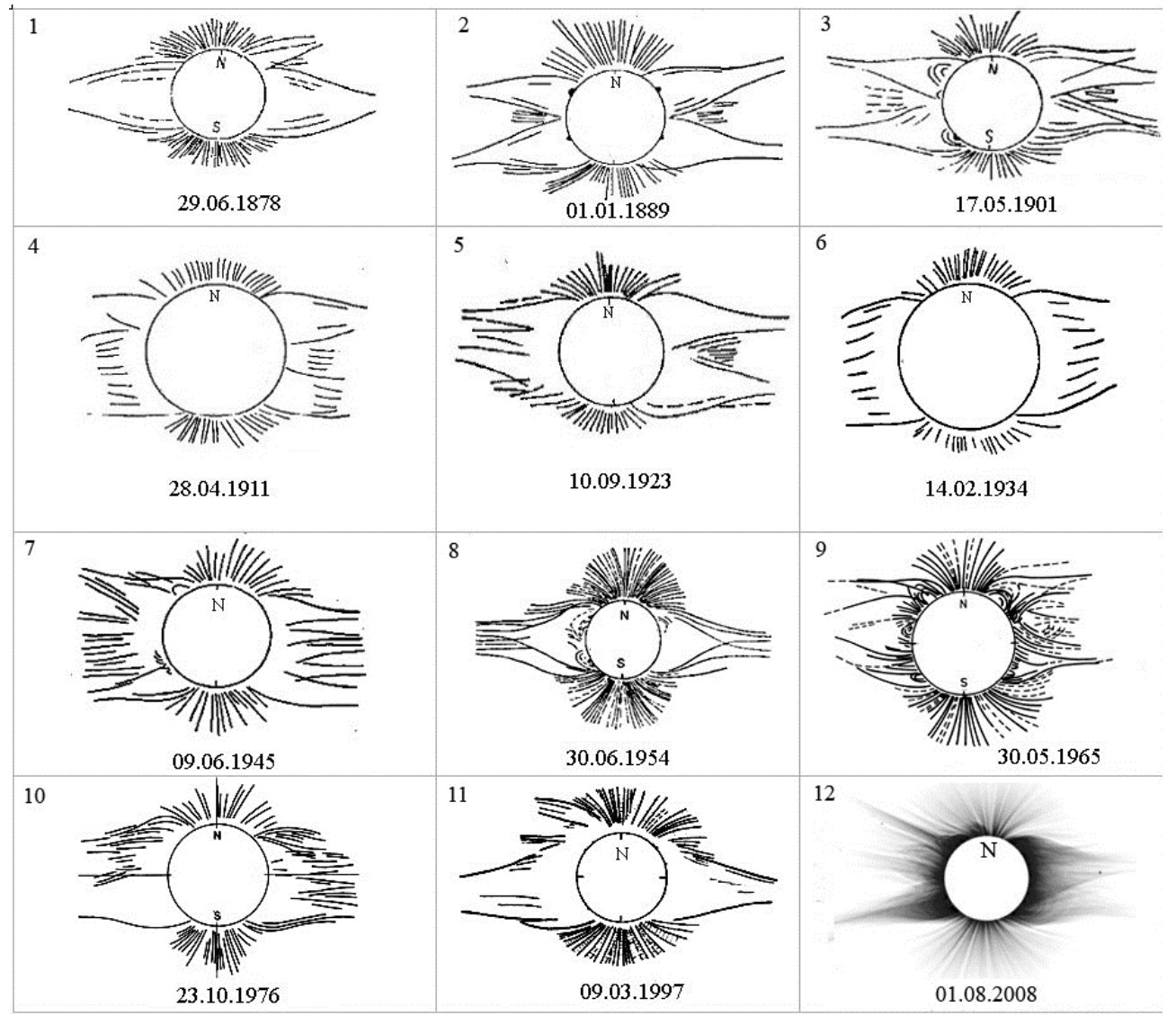

Fig. 3. Eclipses close to the minima of cycles 12--24.

Figure 3 presents the shapes of eclipses for the epochs close to the solar activity minima of cycles 12-24. The calculated values of the parameter $\gamma$ for these eclipses are listed in Table 1. The $\gamma$ parameter varies within $40 \div 100$ degrees. Table 1 also gives the solar cycle phase $\Phi$ (see Eq. 1). One can see that the highest magnitudes of the parameter $\gamma$ occurred during the 


\begin{tabular}{|c|c|c|c|c|}
\hline Cycle No & Date & W & $\Phi$ & Y \\
\hline 12 & 29 June 1878 & 75 & -0.06 & 65 \\
\hline 13 & 21 December 1889 & 88 & 0.03 & 60 \\
\hline 14 & 17 May 1901 & 63 & -0.07 & 80 \\
\hline 15 & 28 April 1911 & 103 & -0.18 & 80 \\
\hline 16 & 10 September 1923 & 77 & 0.04 & 85 \\
\hline 17 & 14 February 1934 & 114 & 0.14 & 97 \\
\hline 18 & 09 June 1945 & 151 & 0.28 & $88^{*}$ \\
\hline 19 & 30 June 1954 & 190 & 0 & 98 \\
\hline 20 & 30 May 1965 & 106 & 0.14 & 90 \\
\hline 21 & 23 October 1976 & 155 & 0,08 & 67 \\
\hline 22 & 22 November 1984 & 158 & -0.35 & $69^{*}$ \\
\hline 23 & 09 March 1997 & 125 & 0.1 & 60 \\
\hline 24 & 01 August 2008 & -- & $\sim 0.1$ & 54 \\
\hline
\end{tabular}

Table 1. Parameters $\gamma$ for the eclipses of cycles $12-24$. W - the amplitude of the Wolf number at the sunspot maximum, $\Phi$ - the phase of solar activity for eclipse date. For cycle 18 and $22 \gamma$ was modified taking the phase of the cycle into account

period 1934--1955. The remaining magnitudes fit the enveloping curve fairly well with a maximum during cycles 17--19 (see Fig. 4). No information on the solar corona structure during the eclipses at the minima of cycles 18 and 22 has been found in the literature. To fill the gaps, the eclipses during the phases of growth or decline of the solar cycle can be used.

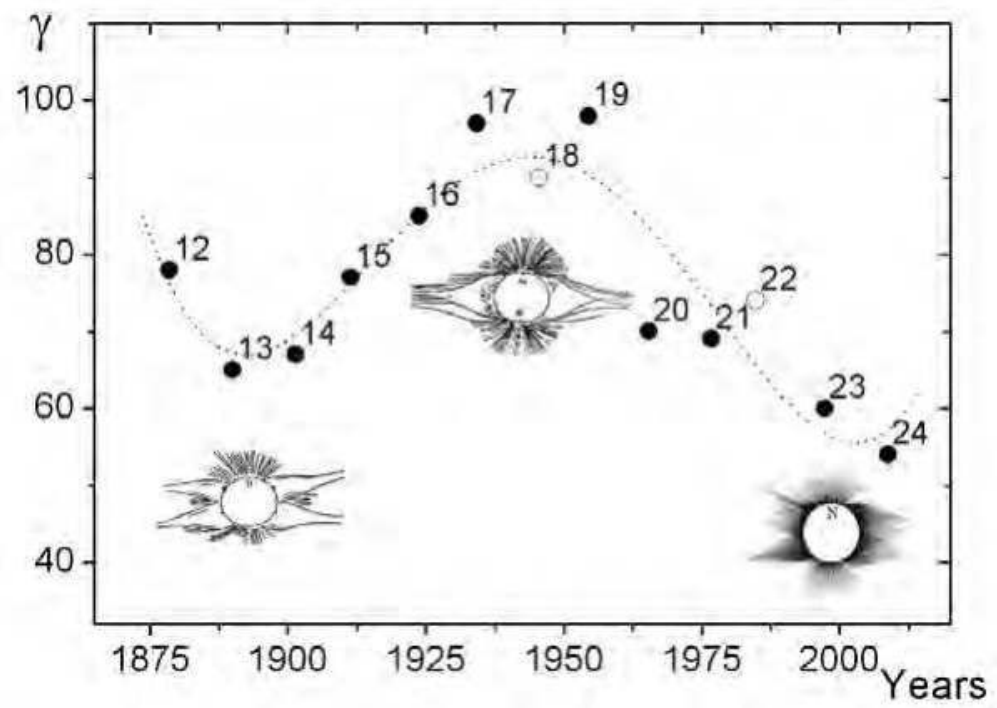

Fig. 4. Distribution of parameter $\gamma$ for the structure of the corona of the minimal type.

Numbers of activity cycles are given. The shape of the solar corona to cycles 13,19 and 24 are present. 
One can see in Table 1 that the eclipses of 1945 and 1984 are rather far from the minimum phase in solar activity. A modified parameter $\gamma^{*}=180-\left(\gamma_{W}+\gamma_{E}\right) \cdot(1-|\Phi|)$ can be introduced for these eclipses. This parameter reduces parameter $\gamma$ to the minimum phase. The application of this procedure is effective for recognizing the shape of the corona close to the minimum activity with the phase $\Phi<0.4$. Figure 4 presents variations in parameter $\gamma$ during the last 13 activity cycles.

\subsection{Long-term changes of coronal shape and geomagnetic disturbance}

The presence of long-term trends in the solar corona structure can be caused by changes in the configuration of the global magnetic field of the Sun. The role of active region formation during a solar activity minimum is not significant. It has long been known that large coronal streamers typically lie above the polarity-inversion lines of the large-scale magnetic field marked by filaments and prominences (Vsekhsvyatskiy et al., 1965). For this reason, investigations of the large-scale corona shape give valuable information on the structure of the large-scale fields during a long time interval. During the activity minimum, the properties of the global magnetic field of the Sun manifest themselves in the most pronounced way.

The magnetic field of the Sun is determined by large-scale structures. The northern and southern hemispheres of the Sun have magnetic fields of opposite polarity. The strength of the polar magnetic field is significantly higher than the fields in middle and low latitudes in the activity minimum period. Along with this, one can conclude from the analysis that assuming that the global solar field configuration is in the form of a dipole structure is probably incorrect. The corona configurations for the eclipses of 1889, 1901, and others correspond instead to the quadrupole form, or to an octopole form if different polarities at the poles are taken into account.

Thus, long-term variations in parameter $\gamma$ should manifest changes in the dipole component during solar activity minima. This hypothesis can be checked using the data on configurations of the large-scale magnetic fields. Figure 5 shows changes in the dipole moment and the envelope drawn through solar activity minima. The data were obtained from the analysis of synoptic $\mathrm{H}$-alpha charts of patterns of polarity inversion lines from (Makarov \& Sivaraman, 1989; Vasil'eva, 2002), and the Kislovodsk Astronomical Mountain Station (http://www.solarstation.ru). The greatest dipole moment corresponded to the minimum of cycle 19 in 1954 . These data were obtained from $H$-alpha synoptic maps. The amplitude of the dipole contributions $l=1$, which determine parameter $A_{1}$ depend on the topology of the large-scale magnetic fields. The method of decomposition is given in the articles (Makarov \& Tlatov, 2000; Tlatov, 2009). On the whole, the envelope of the dipole moment shows similar trends in the changes in the corona shape parameter $\gamma$ (see Figs. 4 and 5).

The growth in the strength of the radial component of the interplanetary magnetic field (Cliver, 2002) determined from the geomagnetic activity index $a a$ is another important problem that has been widely discussed recently. Figure 6 shows variations in the geomagnetic index $a a$. Data were taken from the National Geophysical Data Center (http://www.ngdc.noaa.gov). The first half of the $20^{\text {th }}$ century was characterized by a growth in this index; i.e., the slowly varying component that was especially pronounced 


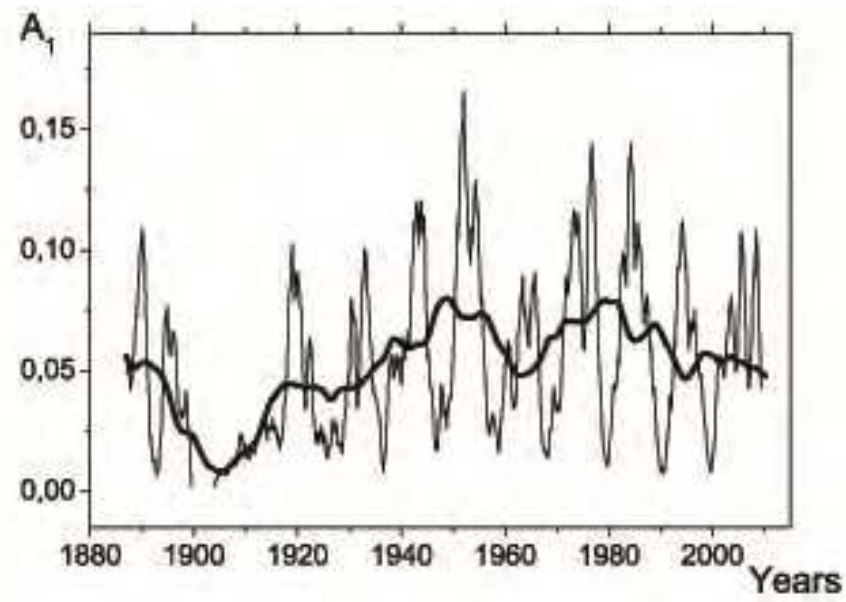

Fig. 5. Variations in the dipole moment derived from synoptic $\mathrm{H}$-alpha charts of the Sun $A(t)=\sum_{m, l=1}\left(g_{l}^{m} g_{l}^{m}+h_{l}^{m} h_{l}^{m}\right), \mathrm{l}=1, \mathrm{~m}=0,1$, where $g_{l}^{m}$ and $h_{l}^{m}$ are the coefficients of the spherical function expansion. The thin line indicates monthly values, and the thick line are 11-year running means.

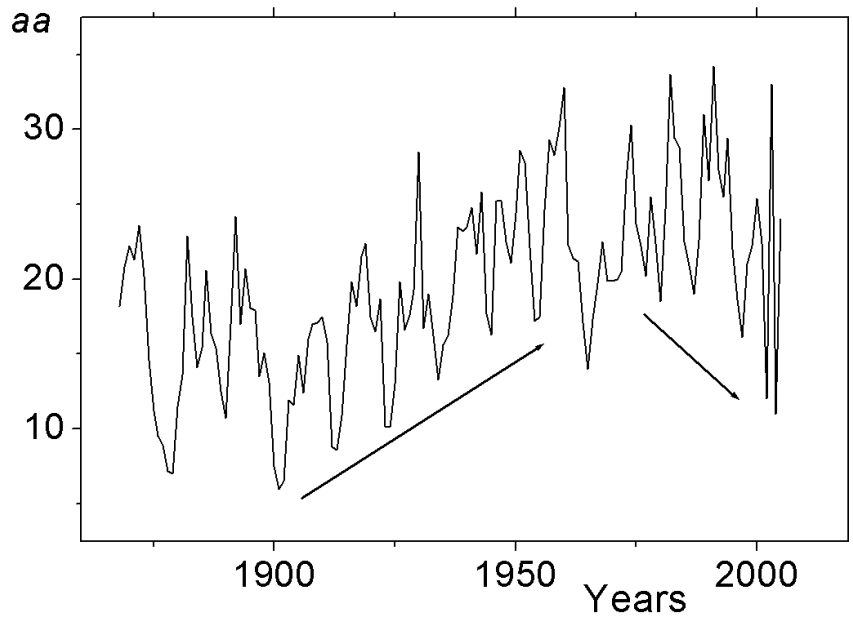

Fig. 6. Annual mean of the geomagnetic indices a from 1868 (according to the National Geophysical Data Center, NGDC), smoothed by 2 years. The arrows mark the index growth in the first half of the 20th century and the index decrease during the last decades for the solar activity minimum epochs.

during the activity minimum epochs grew. During the past decades, a decrease in the $a a$ index during the activity minimum epochs was observed. Probably, this is due to rearrangement of the global magnetic field of the Sun accompanied by changes in the solar corona structure during the minimum epochs. Variations in the geomagnetic index and the 
dipole moment of the large-scale magnetic field of the Sun during the activity minimum epoch are almost identical (see Fig. 7).

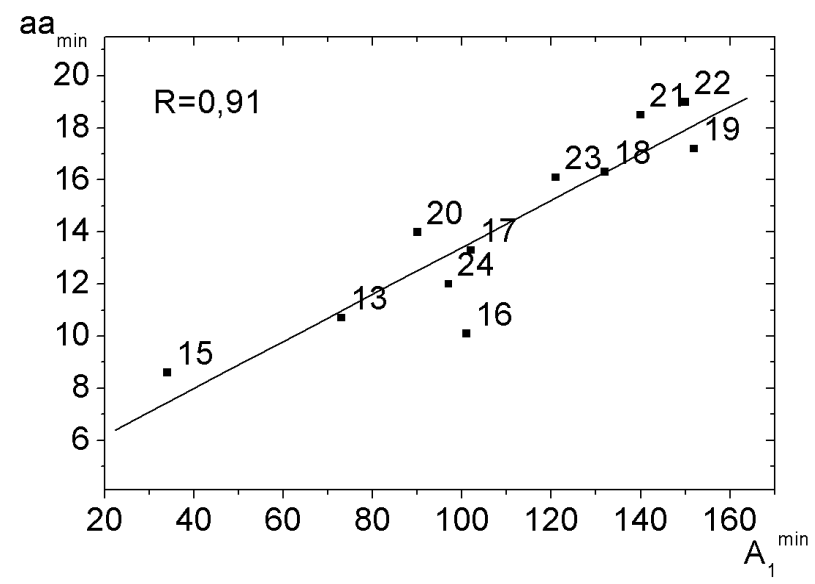

Fig. 7. Relation between the geomagnetic index aa and the magnitude of the dipole moment of the large-scale magnetic field $\mathrm{A}_{1}$ according to Figs. 4 and 5 during the solar activity minimum epoch. Numbers of activity cycles and linear regression are also shown.

We can also test changes deviations of coronal streamers from the radial direction for over 100 years. You can use the catalogs of solar eclipses (Loucif and Koutchmy, 1989; Naegamvala, 1902; Vsekhsvyatskiy et al., 1965).

The corona of the solar minimum is characterized by pronounced polar ray structures and large coronal streamers. Let us introduce index $\gamma$ that characterizes the angle between highlatitude boundaries of the large coronal streamers at a distance of $2 R$. The $\gamma$ index is the sum of the angles at the eastern and western limbs: $\gamma=\gamma_{N}+\gamma_{S}$. In fact, the $\gamma$ index is a simpler version of the corona flattening indices (Nikolskiy, 1955) but it is calculated at height $R$ above solar limb. Figure 4 presents variations in parameter $\gamma$ during the last 13 activity cycles (Tlatov, 2010a).

Thus, analysis of the corona shape has revealed a long-term modulation of the global magnetic field of the Sun. Possibly, there exists a secular modulation of the global solar magnetic field which is most pronounced during the solar activity minimum epoch. During the secular cycle of the global magnetic field of the Sun the relation between the dipole and quadrupole components of the magnetic field changes. The largest amplitude of the dipole component occurred during the interval 1944--1955. At the boundary between the 20th and 21st centuries the solar corona shape and, possibly, the global magnetic field correspond to the configuration close to the octupole one (Fig. 8).

\section{Non-radial spreading of the solar corona structures in solar cycle and variations of the solar wind parameters}

In this section we will consider in detail the physical mechanism linked with variations of the coronal shape and changes of the solar wind parameters. 

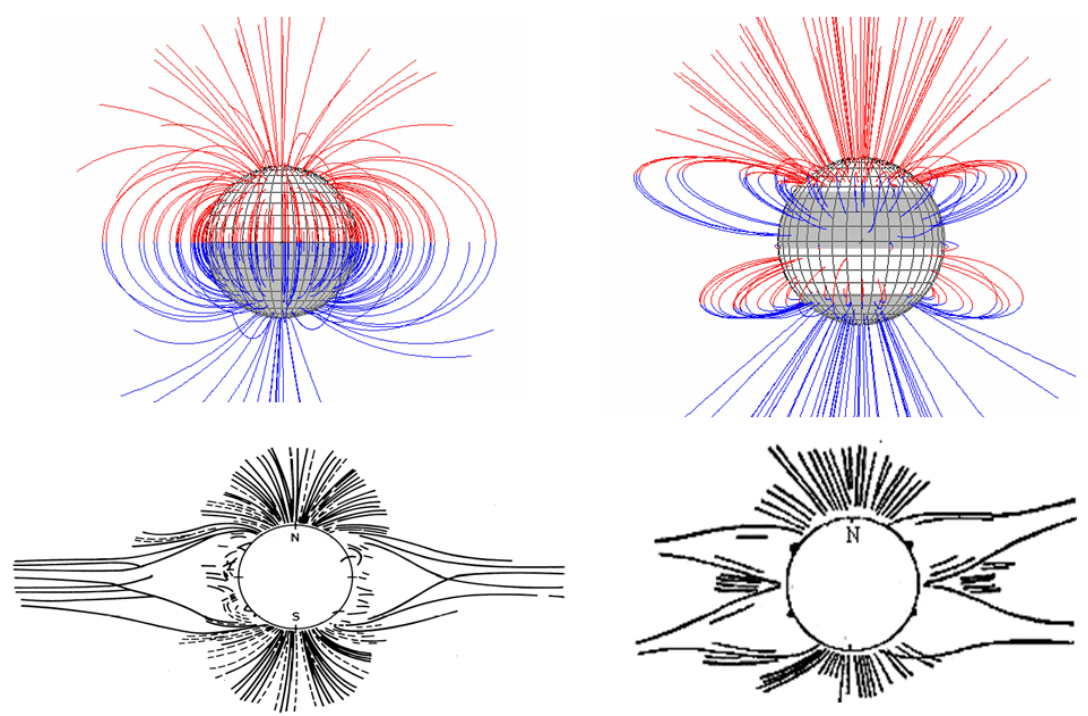

Fig. 8. Configuration of magnetic force lines for the corona of the dipole type (left) and quadrupole or octopole type if different polarities at the poles are considered (right). Regions of negative polarity are darkened. The structure of the corona for the epoch of minimum 13 and 19 activity cycles are also shown.

The solar corona structure corresponds to the configuration of solar magnetic fields. Since the magnetic field of the Sun is subjected to cyclic variations, the corona shape also changes cyclically. The coronal rays are distinctive structures in the solar corona, which propagate at a small angle to the radial direction from the Sun and display the electron density in Kcorona enhanced by the factor of 3 to 10 . The angle $\Delta \theta$ that describes the deviation of the rays from the radial position varies with the phase of the solar cycle and the latitude (Eselevich and Eselevich, 2002; Tlatov 2010b).

The regular observations with the SOHO/LASCO and Mark-III/IV coronagraphs at the Mauna Loa solar observatory make it possible to analyze the structure of the solar corona for the time comparable with the duration of the solar cycle. These data substantially complement extended series of observations of the corona in spectral lines carried out with extra-eclipse coronagraphs, since they make it possible to analyze coronal structures at sufficiently large distances from the solar limb, and also occasional observations of the "white light" corona during total eclipses. The coronagraph -- polarimeter Mark III detected the structure of the solar corona at the heights $\sim 1,15 \div 2,45 R_{\odot}$ in 1980-1999. In 1998, at Mauna Loa observatory the new low-noise coronagraph Mark-IV, with a liquid-crystal modulator of polarization and a CCD, was mounted. To decrease the radial gradient and consequently to increase the contrast, we applied to the Mark data an artificial vignetting function. The LASCO-2 coronagraphic telescope on board of SOHO satellite has been working since 1996 and covers the distance $1.5 \div 6 \mathrm{R}_{\odot}$ above the solar limb. Thereby, here we have analyzed the structure of the corona for 1980-2008 on the basis of the data obtained at ground-based observations with Mark-III/IV coronagraphs and for 1996-2009 with the SOHO/LASCO-2 data. 


\subsection{The identification for the deviation angles of coronal rays}

In order to determine the deviation of coronal rays, we developed a technique of the identification of coronal streamers in two-dimensional images of the corona obtained with SOHO/LASCO-2 and Mark-III/IV in automatic mode. The analysis is based on discrimination of central parts of bright coronal structures propagating, as a rule, at some angle to the radial direction, discrimination of the points of the local maxima, and determination of the parameters of the approximating line section (Figure 9). The procedure included the following stages. Initially, the coordinates of the center and the radius of the Sun were measured in pixels. Further on, we calculated the average limb brightness of the corona for different heights above the solar limb $I(r)$. We considered the regions in which the brightness at a given distance from the limb was no smaller than $0.3 \cdot I(r)$. Then we selected all the points with the brightness exceeding that of the points apart of it by the angle $\pm 1,5^{\circ}$ along the limb. The obtained collections of points, as a rule, represented regions close to the center of brightness of the coronal rays, extended from the limb of the Sun at some angle to the radial direction. For these regions, we inscribed the least-square approximating straight lines. From the parameters of these straight lines we determined the polar angle of the base of a coronal ray $\theta$ and the deviation from the radial direction $\Delta \theta$. Figure 10 presents an example of such identification for a Soho/Lasco-2 image obtained on 2007.03.16. We used for the analysis the deviation angle for these straight lines with respect to the radial propagation. We took into account line sections with the length no smaller than $1.0 \cdot R$ for $\mathrm{SOHO} /$ Lasco-2 images and than $0.5 \mathrm{R}$ for Mark-III/IV images. The selection of rays, the linearity of which can be traced to a fairly large distance, allows to filter out a considerable number of coronal structures associated with eruptive processes. Thereby, we have processed about 4.2.103 images for the time interval 1996-2009 and discriminated approximately $10^{5}$ coronal rays from the Soho/Lasco-2 data and $7 \cdot 10^{3}$ days for the MarkIII/IV data for 1980-2008.

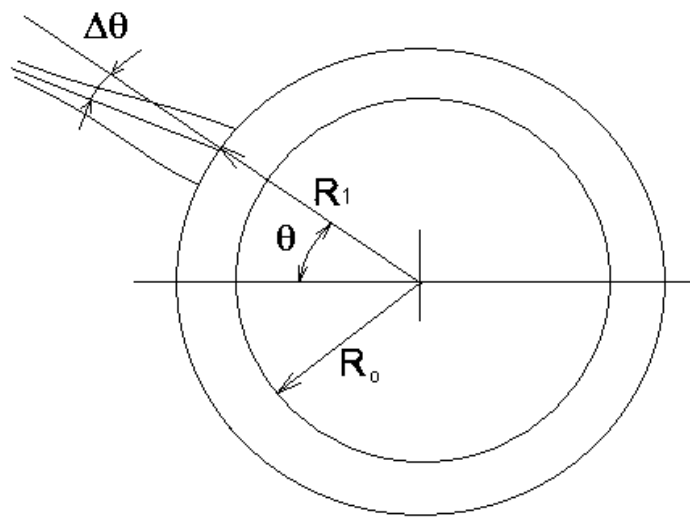

Fig. 9. The determination of deviation angles for coronal rays.

In this analysis, we recorded the coronal beams of various types. Among the rays belonging to the helmet-type, and chains of streamers, rays belonging to the low-latitude and polar regions (Saito et al. 2000, Eselevich E Eselevich, 2002). These rays can be formed over the bipolar and unipolar magnetic structures. There are also dynamic coronal rays, but we 


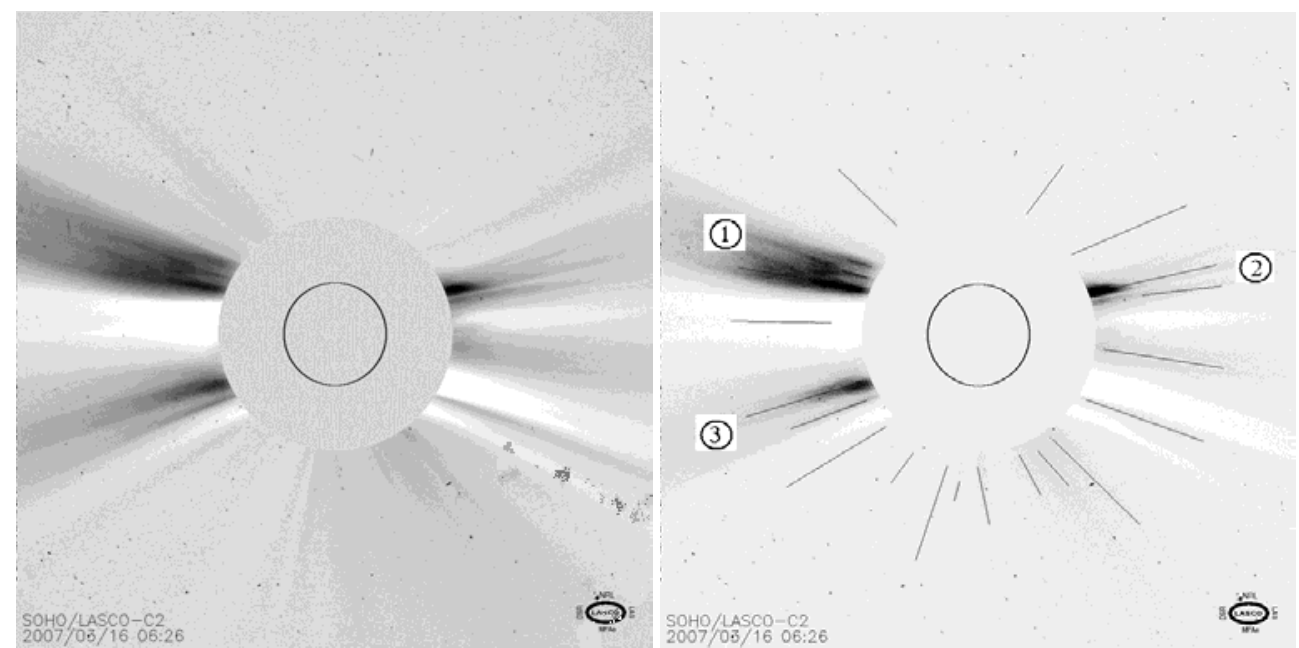

Fig. 10. An example of the identification of the direction of the propagation of coronal streamers for an image of the corona obtained with Soho/Lasco-2 on 2007.03.16.

decided that their number is small enough to that would affect the comparison. Thus Fig. 11 shows that the footpoints rays are usually evident in a few days. In addition to the geometry of the rays in the process of selection, we also recorded the absolute and relative brightness of the rays. This allowed to make a comparative analysis nonradially rays and define their types. In order to define the brightness of rays we calculated the average intensity within the function from the height over the solar limb $I_{\text {avr }}(r)=1 / 2 \pi \int_{0}^{2 \pi} I_{a v r}(r, \alpha) d \alpha$, where $\alpha$ - is a polar angle. Then we defined the average intensity of a ray at the beginning and ending of a segment, approximating the ray $\bar{I}=\left(I_{\text {beg }}+I_{\text {end }}\right) / 2$. The rays with the intensity $\bar{I}>2 \cdot I_{\text {avr }}$, where $I_{\text {avr }}$ was calculated at a height $\left(R_{\text {beg }}+R_{\text {end }}\right) / 2$, were related to bright rays. The rays with the intensity $\bar{I}<1.3 \cdot I_{\text {avr }}$ were conditionally related to non-bright rays. Figure 10 shows the systems of bright rays, that are marked with numbers 1-3. The rays of lower intensity are normally located within the area of high latitudes (4, Fig. 10).

Footpoints of bright coronal rays, obtained under the assumption of linear distribution, as a rule, close to the neutral line (Fig. 11).

Fig. 12 shows the change of nonradiality parameter for bright rays $\Delta \theta_{b r}$ associated with helmet-rays and soft rays $\Delta \theta_{\text {low }}$, which are typical for the chain of coronal streamers from the unipolar regions. Rays of varying brightness show close cyclical course of the parameter $\Delta \theta$, although the degree of nonradiality for bright rays is slightly less: $\Delta \theta_{b r}=1.46+0.47 \cdot \Delta \theta_{\text {low }}$, are correlated with $r=0.79$. Rays of different latitudinal zones also show a close behavior (Fig. 13). Relationship between the parameter $\Delta \theta$, for rays of the equatorial and polar zones is the following: $\Delta \theta_{p o l}=4.1+0.84 \cdot \Delta \theta_{e q} ; r=0.89$. Thus, the nonradial parameter $\Delta \theta$ cycle exists for the various types of coronal rays. 


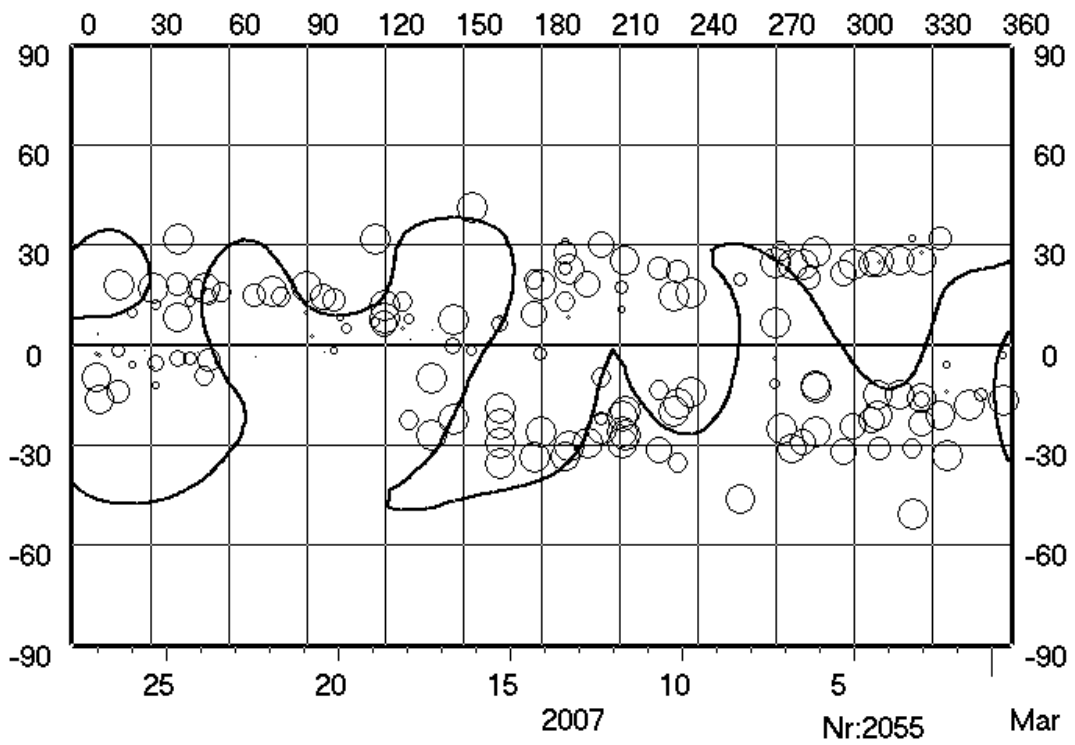

Fig. 11. Example of synoptic maps for Carrington rotation N2055 which marked the neutral line, calculated at an altitude $\mathrm{R}=1.9 \cdot \mathrm{R}_{\odot}$ according to the Wilcox Solar Observatory. Presents the footpoints of bright coronal rays.

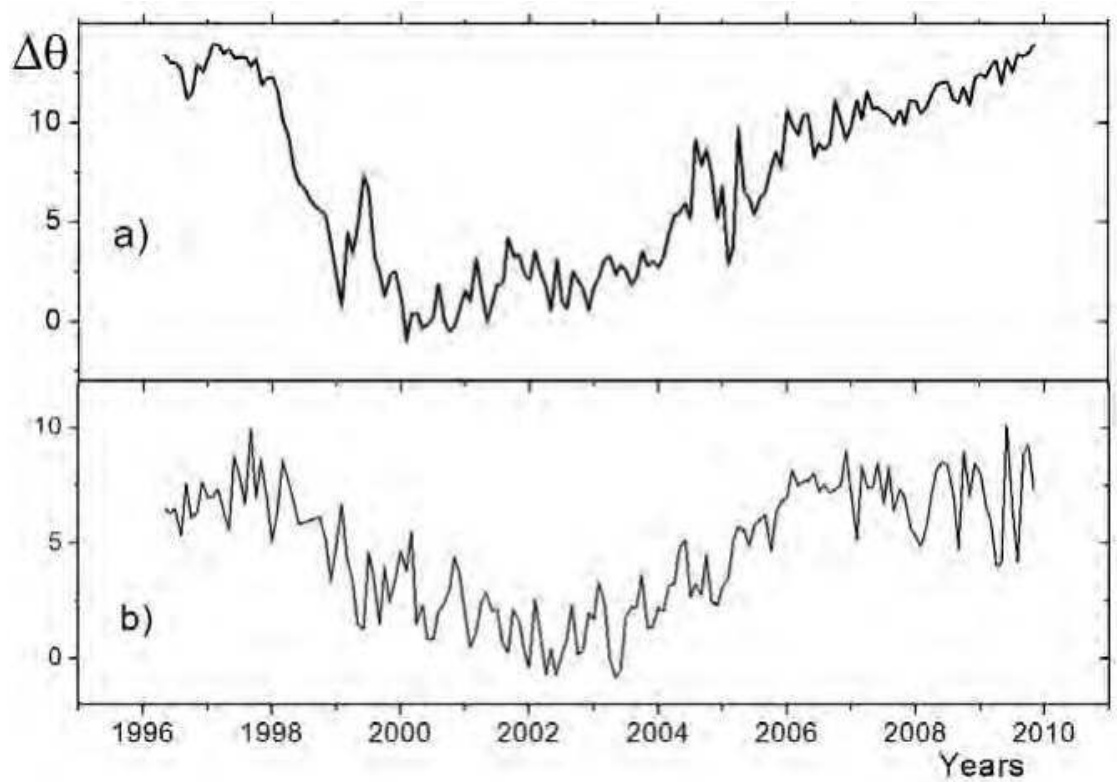

Fig. 12. The monthly-averaged deviation from the radial direction $\Delta \theta$ derived from the $\mathrm{SOHO} /$ Lasco-2 data a) for coronal rays of low intensity $I<1.3 \cdot I_{\text {avr }}$ b) for bright coronal rays $\left(I>2 \cdot I_{\text {avr }}\right)$. 


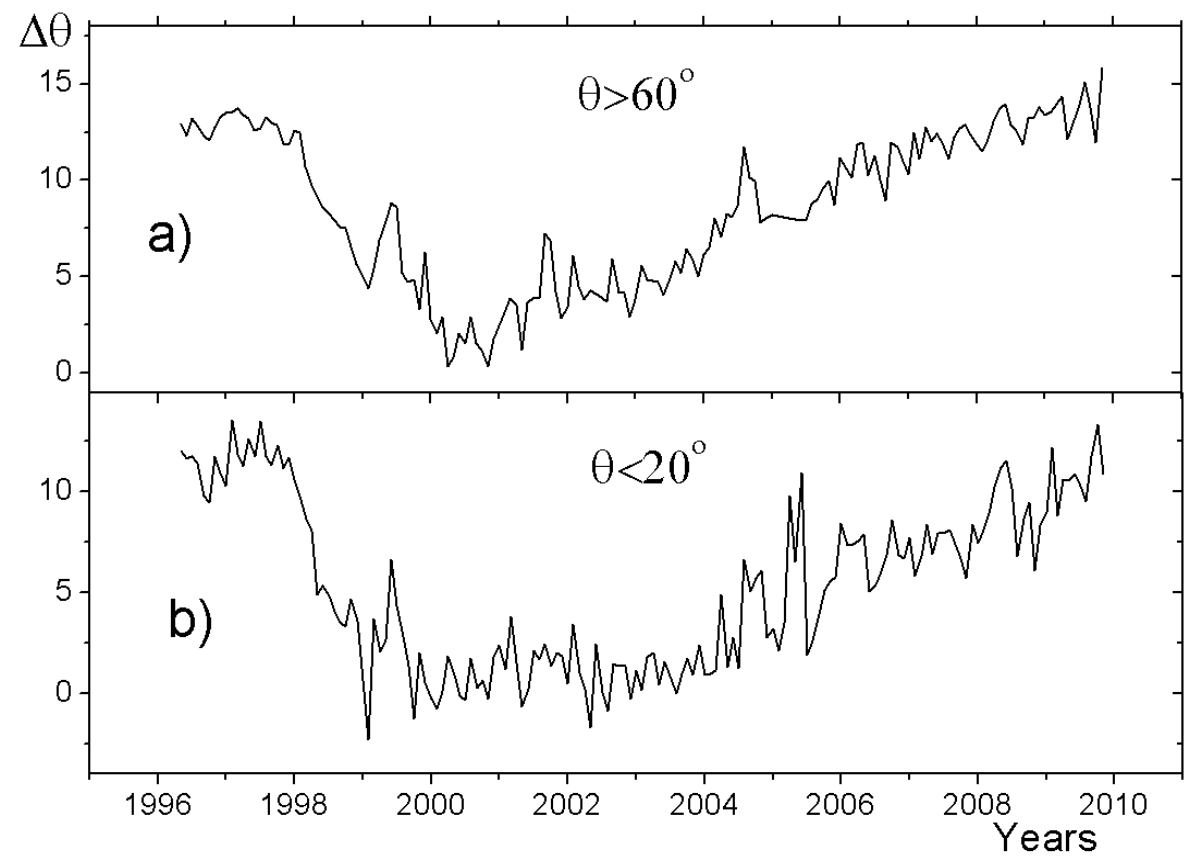

Fig. 13. The monthly-averaged deviation from the radial direction $\Delta \theta$ derived from the $\mathrm{SOHO} /$ Lasco-2 data a) for the polar rays b) for the rays of the equatorial zone.

\subsection{Connection between the non-radial coronal shape and the solar wind parameters}

The analysis of the SOHO/Lasco-2 data for 1996-2009 indicates that the deviation angle $\Delta \theta$ varies with the 11-year cycle of the activity, reaching the maximum values at the minimum of the activity. Figure 14 presents the time-latitude diagram for $\Delta \theta$ variations. In the vicinity of the equator, the deviation of the rays from the radial direction reaches the minimum. Negative angles were seen in the northern hemisphere in 2002-2004 and in the southern hemisphere in 2000-2003, at the latitudes smaller than 30. The coronal rays at the minimum of the activity and the phases of the decline are, as a rule, turned towards the solar equator. Only rather low-latitude coronal rays at the maximum of the activity slightly deviate towards the poles (Fig. 14). The largest deviation from the radial direction is seen at the minimum of the activity at the latitudes $30 \div 60^{\circ}$. At the time of the maximum of the activity and the polarity reversal of the solar magnetic field the rays are directed either parallel to the equator or slightly deviate towards the poles. Individual fluctuations of the angles of deviation of the rays are seen in different latitude zones (Figure 13), which indicates the general type of the perturbations of coronal structures.

Figure 15 presents the monthly averages for the deviation, averaged along the entire limb for all types of coronal rays. For comparison, the graph also shows the variation of the angle of tilt angle $\tau$ of the heliospheric current sheet (HCS) (Hoeksema \& Scherrer, 1986). Tilt angle HCS is calculated in a potential assumption of this photospheric magnetic field for $R=3.25 \cdot R_{0}$. Between these parameters there is the ratio: $\Delta \theta=0.49+0.2 \cdot \tau ; r=0.91$. 


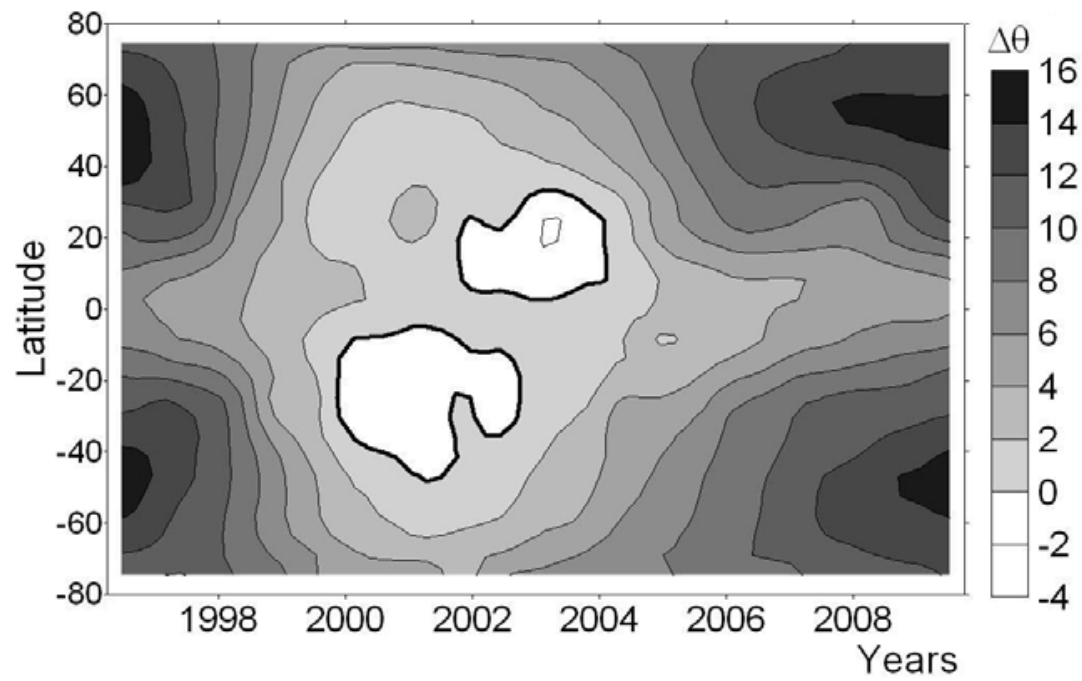

Fig. 14. The latitude and time distribution of the angles of the deviation of coronal rays from the radial direction in the time interval 1996-2009 derived from the SOHO/Lasco-2 data. The regions of the deviation of the rays towards the equator are darkened. The levels are indicated at the intervals of 2 degrees; the level corresponding to $\Delta \theta=0^{\circ}$ is shown by the thick line.

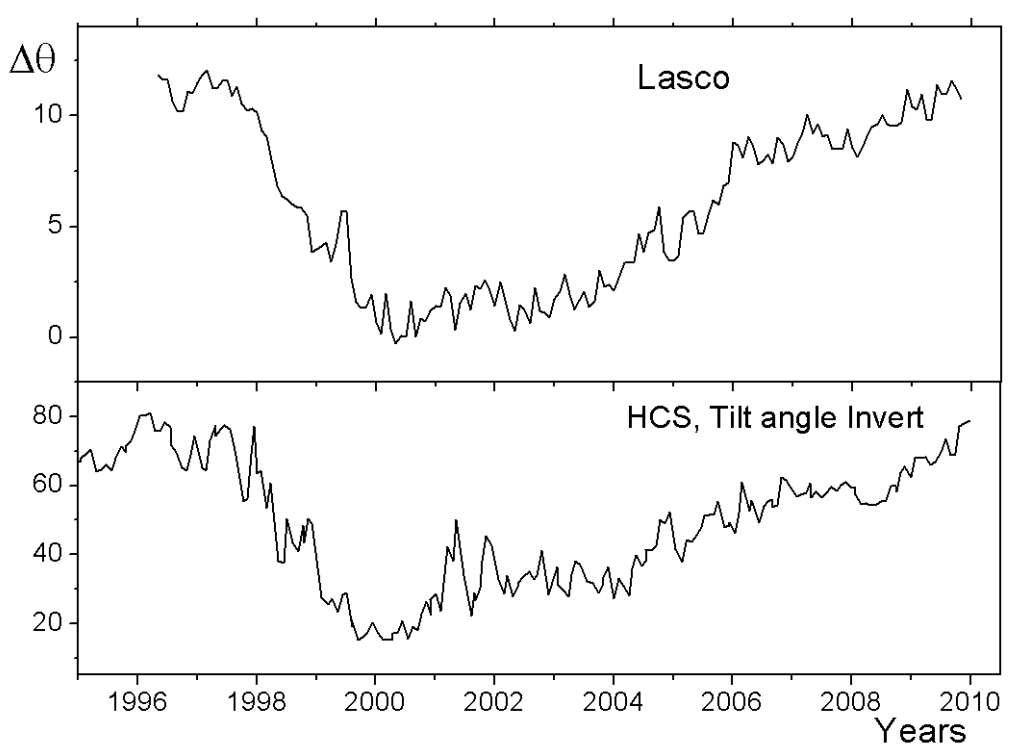

Fig. 15. Comparison between the deviation $\Delta \theta$ of coronal rays from SOHO/Lasco-2 (top panel) and the inverted tilt angle of the heliospheric current sheet according to WSO (http://wso.stanford.edu). 
These conclusions are also confirmed by the analysis of the structure of the solar corona at the heights $1.2 \div 2.5 \mathrm{R}$ based on the MLSO Mark-III/IV data. Figure 16 presents the angle $\Delta \theta$ for the latitude zone $\pm 30^{\circ}$ in the time interval 1980-2008. For the period 1996-2008 angle $\Delta \theta$ according to the coronagraph Mark was slightly lower than according to Lasco/C2: $\Delta \theta_{\text {Mark }}=2.55+0.66 \cdot \Delta \theta_{\text {Lasco; }} r=0.95$. The nonradial variation $\Delta \theta$ according to Mark telescope during 1980-2008 change of the parameter also has a good correlation with the angle of tilt angle of HCS $r \sim 0.8$ (Fig. 16).

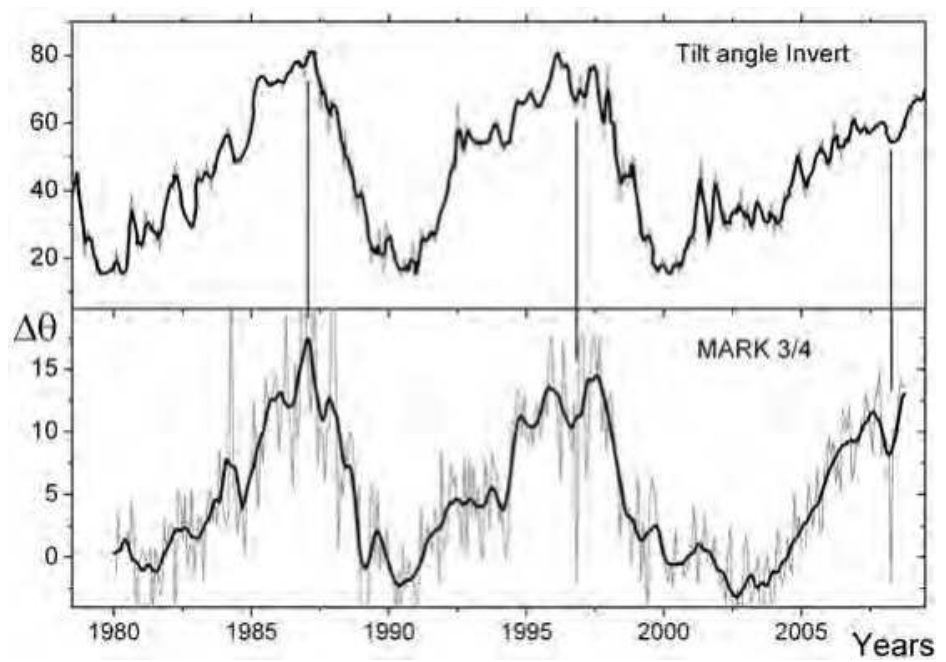

Fig. 16. The comparison between the deviations $\Delta \theta$ of coronal rays located in the middlelatitude zone $\pm 30^{\circ}$, from the MLSO- MarkIII/IV data (bottom panel) and the inverted tilt angle of the heliospheric current sheet according to the WSO (top panel). The values are smoothed for 6 months.

Figure 17 presents the latitude distribution for the $\Delta \theta$ angle for the time of the minimum of the 23-rd cycle. The maximum deviation is seen at the latitudes $\sim 40-50^{\circ}$, which corresponds to the data obtained with the Lasco telescope (Eselevich \& Eselevich, 2002).

The deviation of coronal rays from the radial direction $\Delta \theta$ related to the solar activity cycle may affect substantially the formation of the solar wind and geomagnetic perturbations. From geometrical consideration, in Figure 1 the flux of the solar wind at the distance $r$ is related to that at the distance $R_{1}$, where the current lines become straight, as follows: $n v / n_{1} v_{1}=1+(\theta-\Delta \theta) \cdot\left(r-R_{1}\right) / \theta \cdot R_{1}$, for $\Delta \theta<\theta$. Assuming that at the years of the minimum activity $\theta=40^{\circ}$ and $\Delta \theta=20^{\circ}$ (Figure 9 ), we obtain that the ratio $n v / n_{1} v_{1}$ at the distance $1 \mathrm{AU}$ increases roughly twice compared to the case of radial expansion.

Figure 18 presents the graph of the deviation angle in comparison with the indexes connected with parameters of a solar wind. In the years of the maximum activity, the influence of active regions and flares is substantial. However during a minimum of activity the deviation $\Delta \theta$ to a plane of solar equator can be one of the reasons of delay of a solar wind speed (Figure 18c), and to modulate indexes of geomagnetic activity aa (Figure 18d) and cosmic rays intensity (CRI) (Figure 18b). 


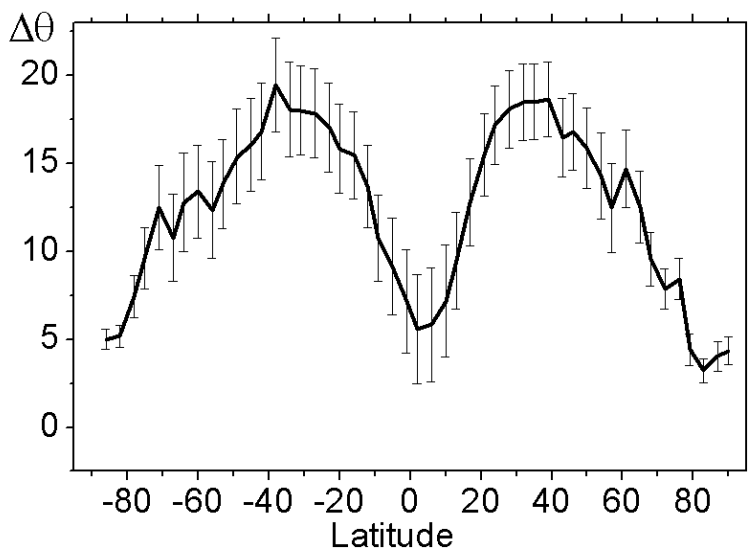

Fig. 17. The variations of the angle $\Delta \theta$ as a function of the latitude, obtained from the MLSOMarkIII data at the minimum of the 23-rd cycle of the solar activity (1996-1997).

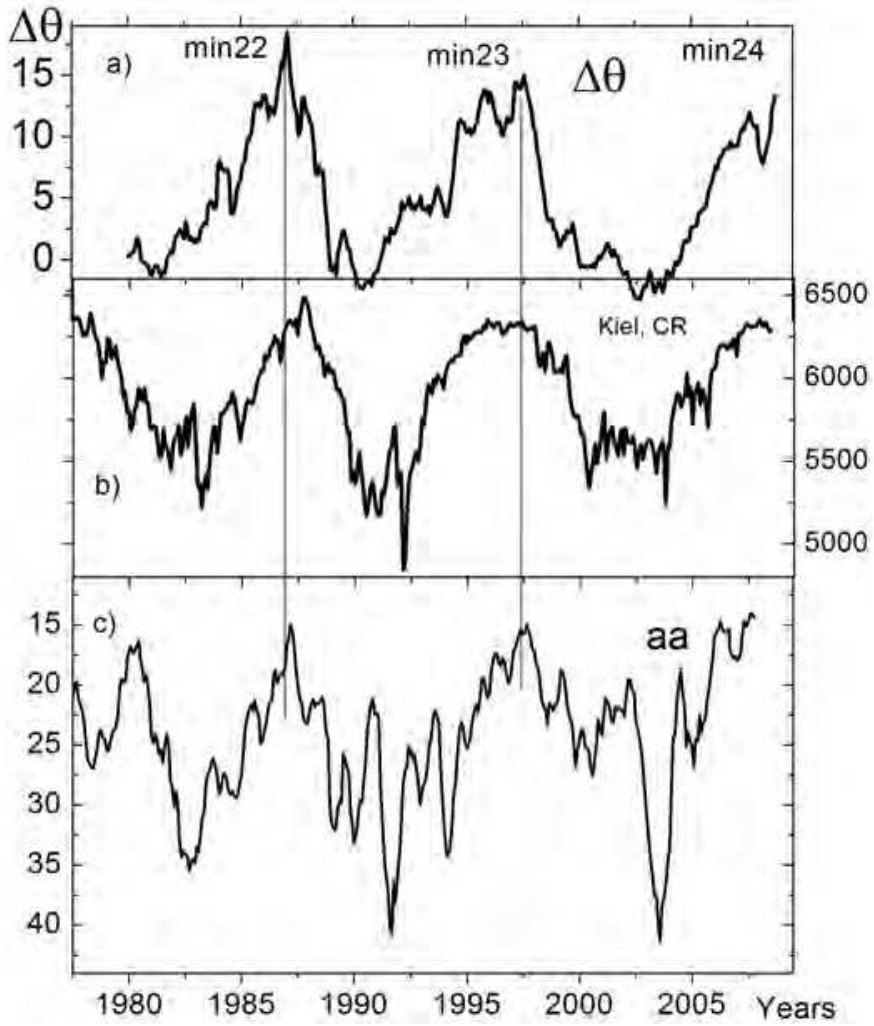

Fig. 18. The comparison between a) the deviations of coronal rays $\Delta \theta$; $b$ ) data of Kiel cosmicray intensity (arbitrary units, monthly means); d) the geomagnetic index $a a$. The data are smoothed with a sliding window technique for 6 months. 
The similarity of the behavior of the shape of the coronal rays with the indices of geomagnetic activity and the radial flux give evidence concerning the connection between the large-scale organization of the corona and solar wind parameters. But is it possible to establish the link directly between the coronal shape and solar wind parameters, measured from the Earth? Data bases OMNI1 and OMNI2 (http://nssdc.gfc.nasa.gov/omniweb) contain the information concerning hourly average value of key parameters of the solar activity, interplanetary atmosphere and geomagnetic disturbance since 1964 . We collated changes of $\Delta \theta$ parameter with solar wind parameter $\beta=8 \pi n k T / B^{2}$ and magnetic Mach number $M_{a}=v / v_{a}=v \sqrt{\mu \mu_{o} \rho} / B$ (Fig. 19). In minimal activity epoch there is a correspondence between the non-radial parameter $\Delta \theta$ and solar wind parameters. Comparing parameters $\beta$ и $M_{a}$ we can make a conclusion that non-radial corona influences the relation $\rho / B^{2}$ of the solar wind, which grows in minimum solar activity and therefore magnetic field in minimal activity squeezes the solar wind flux towards helio-equator. These results complement the findings of in comparison with the previous three minima, this solar minimum has the slowest, least dense, and coolest solar wind, and the weakest magnetic field (Jian et al., 2011).

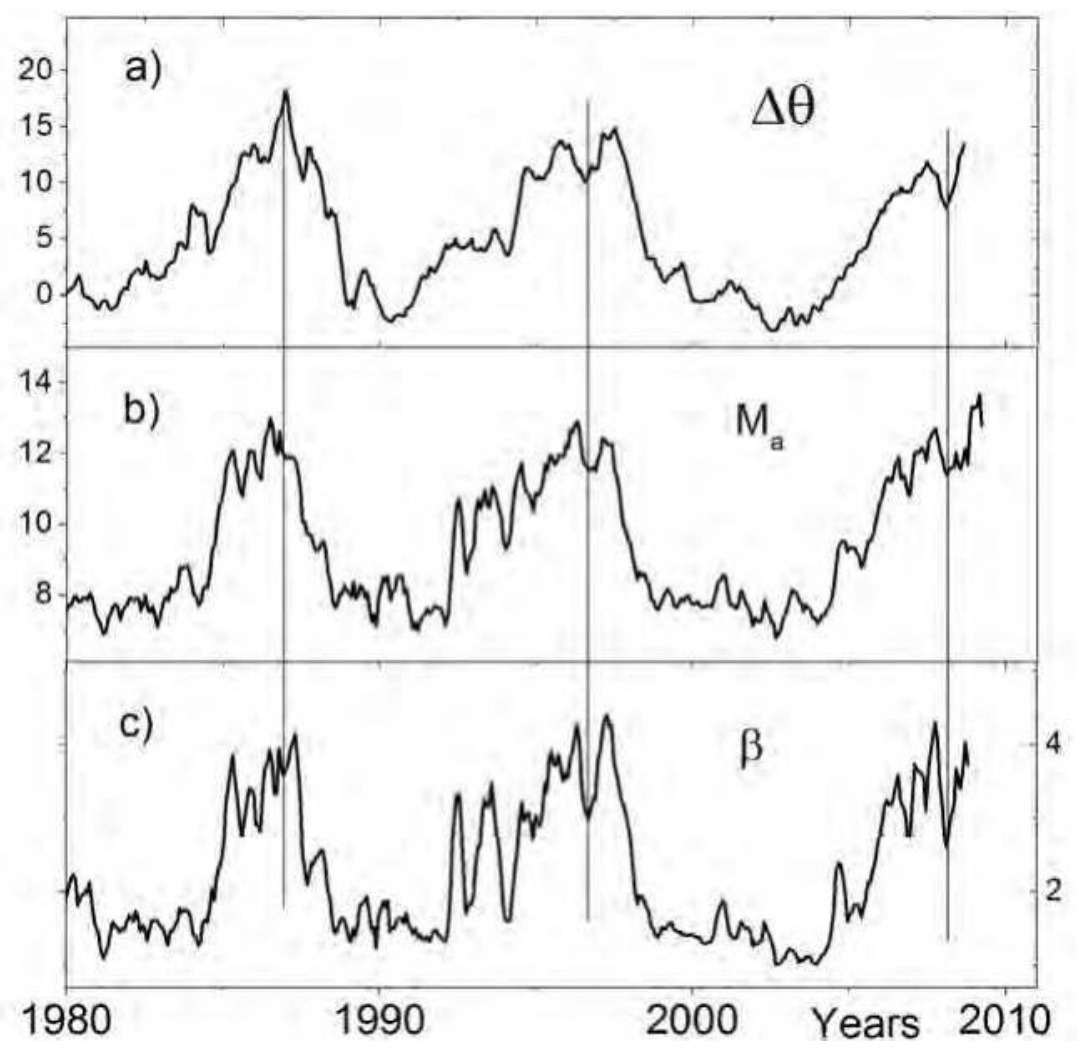

Fig. 19. The comparison between a) the deviations of coronal rays $\Delta \theta$ with the solar wind parameters according to OMNI2 database b) Alfven mach number Ma and c) Plasma beta $\beta$. Data of the solar wind parameters were are smoothed on 20 Bartels rotations. 


\section{Conclusion}

Nowadays to prognose the solar wind parameters empirical models are used (Wang, Y.-M., \& Sheeley, 1990; Arge \& Pizzo, 2000), they are based on the partial understanding of the physical processes taking place on the Sun. At the heart of these ideas there is a hypothesis about the radial character of the solar wind spreading. The details of the streams formation of the solar wind are complicated and are the subject of many researches. Nowadays there aren't any exact mathematical models of the physical processes influencing the formation of the solar wind flux.

In our research we pay attention to the fact that in minimal activity the conditions of forming the solar wind can depend on the configuration of the large-scale field and the shape of the solar corona. The configuration of the global magnetic field in minimal activity corresponds to the low harmonics of decomposition according to the Legendre polynomials $l=1,2,3$.

The link between the coronal shape, geomagnetic disturbances and solar wind parameters can be clearly seen in 11-year activity cycle. Global magnetic field can lead to non-radial spreading of the coronal streamers and probably, to the spreading of the solar wind. In minimal activity when the value of the global field reaches its maximum, coronal rays are deflected aside from the helio-equator.

Thus, analysis of the corona shape has revealed a long-term modulation of the global magnetic field of the Sun. Possibly, a secular modulation exists of the global solar magnetic field that is most pronounced during the solar activity minimum epoch. During the secular cycle of the global magnetic field of the Sun, the relation between the dipole and octopole components of the magnetic field changes. The largest amplitude of the dipole component occurred during the interval 1944--1955. At the turn of the 19th to 20st and 20th to 21st centuries the solar corona shape and, possibly, the global magnetic field correspond to the configuration close to the octopole one (see Fig. 8).

The period of variation in the corona's shape during the epoch of minimal activity is about $100 \div 120$ years (see Fig. 4), which is close to the Gleissberg cycle for the sunspots, but probably precedes it to some extent in phase (Hathaway, 2010, Fig. 34).. The maximum of the secular variation in the global magnetic field of the Sun occurred before cycle 19 and preceded the sunspot activity maximum. This allows us to put forward the hypothesis that secular variations in the solar activity are caused by secular modulation of the global magnetic field of the Sun. Another conclusion of this work is the supposition that the slowly changing component of the geomagnetic activity derived from the data on the a index from changes in the dipole component of the large-scale field of the Sun (Figure 7).

The link between the coronal shape, geomagnetic disturbances and solar wind parameters can be clearly seen in 11-year activity cycle. Global magnetic field can lead to non-radial spreading of the coronal streamers and probably, to the spreading of the solar wind. In minimal activity when the value of the global field reaches its maximum, coronal rays are deflected aside from the helio-equator. Here with lines of force influence the elapsing flux of the solar wind, squeezing it towards the ecliptic plane (Fig. 19). It is possible that long-term changes of geomagnetic disturbances that do not tend to zero even in the minimum activity are not conditioned this very deflection of the solar wind. 
The awareness of the variations of the angle $\Delta \theta$ with the phase of a cycle is important for theoretical models describing the structure of the corona and the geometry of the magnetic field above the solar limb (Wang, 1996). The applied aspect of the studies for the deviations of coronal streamers, along with the plasma flows in the solar wind, is also of great importance, since the deviation may affect the geo-efficiency of the solar wind impact. The presence of long-term trends in the solar corona structure can be caused by changes in the configuration of the global magnetic field of the Sun. The non-radial propagation of the solar wind may explain the relationship between geomagnetic indices detected during the minima of activity and the amplitude of a subsequent solar cycle (Ohl, 1966). Indeed, the relation between the amplitude of the angles $\Delta \theta$ at the minima of the 22-th - 24-th cycles is close to that for the index of the large-scale magnetic field of the Sun in these times (Tlatov, 2009), and this field is one of prognostic indices of the solar activity. Thereby, we may suggest a link between the large-scale magnetic field and the deviation of coronal rays towards the equator, which in turn affects the level of geomagnetic indices at a solar minimum as well as solar-terrestrial relations.

The deviation coronal streamers $\Delta \theta$ occur as the cyclic process appreciable at all solar latitudes (Figure 13,14). Probably, it is connected with global processes, for example hemispheric current layer (Filippov, 2009).

In the analysis we have considered the various structures, including possibly producing a unipolar coronal streamer belt (called' 'chains of streamers" or "streamer belt without a neutral line" by (Eselevich et al., 1999) as well as the bipolar streamer belt (Zaho \& Webb, 2003). These streamers vary in brightness and location of footpoint. Our analysis of separation of their brightness and latitude showed that cyclic changes in the nonradial parameter $\Delta \theta$ exist for various types of coronal streamers. The good agreement between the change in time for parameter $\Delta \theta$ change the angle of inclination of HCS, suggest that the parameter of nonradiality, is possibly conditioned by the existence of current in the heliospheric current layer. The current in the HCS can be estimated from the parameter $\Delta \theta$.

In the epoch of the 18-19 activity cycles in the middle of the last century the shape of the solar corona in its minimal activity mostly corresponded to the corona of the minimal type, that is - coronal radiance spreaded along helio-equator (Fig. 3). Just in that period the highest level of geomagnetic activity was observed (Fig. 6), what is more, not only during the years of solar spots maximum, but also in the minimal activity. We can assume that during that period global magnetic field of the Sun most effectively squeezed the solar wind flux towards the heio-equator plane, which led to such an effect.

Variation changes of the low and high harmonics order are illustrated on figure 20. Here the amplitudes of the harmonics were smoothed by the "running window" of 11 years long. One introduces amplitudes of axisymmetric mode for dipole and quadruple $(1=1,2 ; m=0)$ and for harmonics, characterizing sector structure of the higher harmonics $(1>3 ; \mathrm{m}>0)$. One can notice that changes in time within the amplitudes of these two types occur in antiphase. One should also notice that axisymmetric modes $(\mathrm{m}=0)$ show that magnetic structures of the opposite polarities are situated on different sides of the equator. On the contrary, the amplitude growth of the sector harmonics shows that opposite signs structures are drawn along meridians. The amplitudes of the axisymmetric harmonics reach their maximum in 


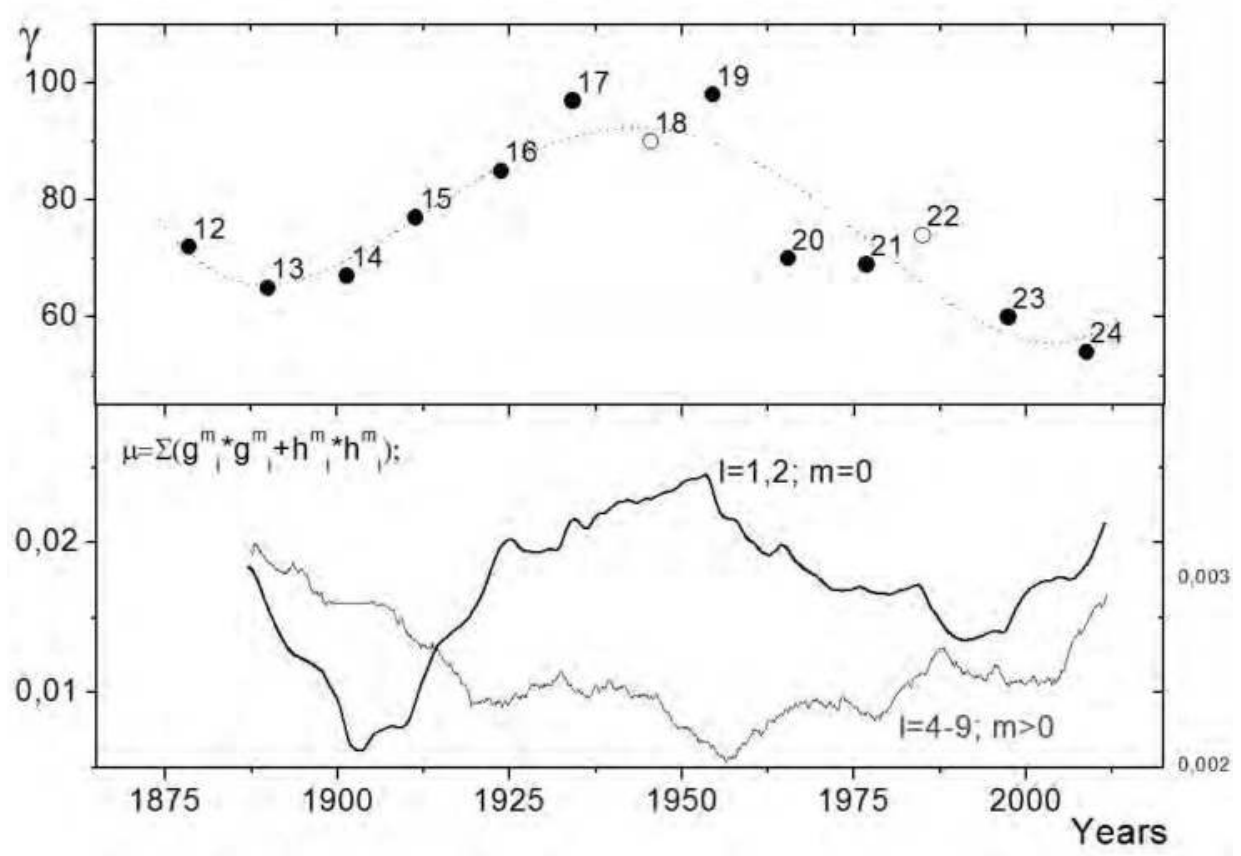

Fig. 20. Variation changes of the low and high harmonics order are illustrated on figure 20. Here the amplitudes of the harmonics were smoothed by the "running window" of 11 years long. Bottom panel introduces amplitudes of axisymmetric mode for dipole and quadruple $(1=1,2 ; \mathrm{m}=0)$ and for harmonics, characterizing sector structure of the higher harmonics $(1>3 ; \mathrm{m}>0)$. At the top changes of parameter $\gamma$ form the solar corona.

the epoch of years 1940-1950, while sector harmonics were minimal at that moment. Such allocation corresponds to the Babcock hypothesis concerning magnetic fields generation. In fact, reconnection of the opposite through equator fields causes mutual destruction of the fields of the leading polarity, and it should cause the solar activity growth. But on the contrary, reconnection of the magnetic field along the meridians does not promote the creation of global field of dipole type and leads to the decline of solar activity level. 
Consequently, in the epoch of big solar activity cycles the growth of geomagnetic activity takes place not only due to the result of active processes increase, but also under the influence of solar wind's formation by the global magnetic field of the Sun in activity minimums. The following fact contributes to this - before the high cycles of activity the intensity of global magnetic field is also high (Fig. 5). It is possible that age-long cycle of changes of solar wind parameter and geomagnetic activity, close to Gleissberg cycle is caused not only by the level of activity, but also by long-term changes of the large-scale magnetic field.

Thereby, we may suggest a link between the large-scale magnetic field and the deviation of coronal rays towards the equator, which in turn affects the level of geomagnetic indices at a solar minimum as well as solar-terrestrial relations.

\section{Acknowledgements}

This paper was supported by the Russian Fund of Basic Researches and Program of the Russian Academy of Science.

\section{References}

Arge, C. N., \& Pizzo, V. J. (2000). Improvement in the prediction of solar wind conditions using near-real time solar magnetic field updates, J. Geophys. Res.,Vol. 105,pp. 10465-10480.

Cliver, E. W. \& Ling, A. G. (2002). Secular change in geomagnetic indices and the solar open magnetic flux during the first half of the twentieth century, J. Geophys. Res., SSH 11$1,107$.

Eselevich, V. G., \& Eselevich, M. V. (2002). Study of the nonradial directional property of the rays of the streamer belt and chains in the solar corona, Solar Phys., Vol. 208, pp.

Eselevich, V. G., Fainshtein, V. G. \& Rudenko, G. V., (1999). Study of the structure of streamer belts and chains in the solar corona, Solar Phys. , Vol. 188,pp. 277-297.

Filippov, B. P. (2009), Non-radial coronal streamers in the course of the solar cycle, Astronomy Reports, Vol. 53,pp. 564-568.

Ganskiy, A. P. (1897). Die totale sonnenfinsterniss am 8. august 1896, Proc. Royal. Akad. Sci. (in Russian), Vol. 6, 251.

Golub, L. \& Pasachoff, J. M. (2009). The Solar Corona, second edition, (Cambridge University Press).

Gulyaev, R. A. (1998). New results of observations of eclipsing the solar corona, in Proceedings of the Conference "'A New Cycle of Solar Activity: Observations and Theoretical Aspects'" (in Russian), ed. A. V. Stepanov, (Pulkovo, St.Petersburg), pp. 61-66.

Hathaway, D. H. (2010). The Solar Cycle, Living Rev. Solar Phys., 7, (http://solarscience.msfc.nasa.gov).

Hoeksema, J. T. \& Scherrer, P. H. (1986). An atlas of photospheric magnetic field observations and computed coronal magnetic fields: 1976-1985, Sol.Phys., Vol. 105,pp. 205-211. 
Jian, L. K.; Russell, C. T. \& Luhmann, J. G. (2011). Comparing Solar Minimum 23/24 with Historical Solar Wind Records at 1 AU, Solar Phys., Doi 10.1007/s11207-0119737-2.

Loucif, M. L. \& Koutchmy S. (1989). Solar cycle variations of coronal structures, Astronomy and Astrophysics Supplement Series, Vol. 77, pp. 45-66.

Makarov, V. I. \& Sivaraman, K.R. (1989). Evolution of latitude zonal structure of the largescale magnetic field in solar cycles, Solar Phys., 119, 35-44.

Makarov, V. I. \& Tlatov A. G. (2000). The Large-Scale Solar Magnetic Field and 11-Year Activity Cycles, Astron. Rep., Vol. 44, pp. 759-764.

Meyer-Vernet N. (2007). Basics of the Solar Wind, ISBN 978-052-1814-20 Cambridge University Press

Naegamvala, K. D. (1902). Report on the total solar eclipse of January 21-22, 1898, (Bombay), 49.

Nikolskiy, G. M. (1955). Forecast form the solar corona on June 20, 1955, Astron. Circular, $160,11-12$.

Ohl, A. I. (1966). Soln. Danie, N 12, 84.

Parker E. (1958). Dynamics of the Interplanetary Gas and Magnetic Fields, The Astrophysical Journal, Vol. 128, pp. 664-676

Pasachoff, J. M., Ružin, V., Druckmüller, M., Druckmülerovä, H., Belik, M., Saniga, M., Minarovjech, M., Markovä, E., Babcock, B. A., Souza, S. P. \& Levitt, J. S. (2008). Polar Plume Brightening During the 2006 March 29 Total Eclipse, ApJ, Vol. 682, pp.

Pneuman, G. W. \& Kopp, Roger A. (1971). Gas-Magnetic Field Interactions in the Solar Corona , Solar Phys., Vol. 18, pp.258-270

Saito, T., Shibata, K., Dere, K.P. \& Numazawa, S. (2000). Non-Radial Unipolar Coronal Streamers in Magnetically High Latitudes and Radial Bipolar Streamers at the Magnetic Equator of the Sun, Adv. Space Res., Vol. 26,pp. 807-810.

Tlatov, A. G. (2009). The Minimum Activity Epoch as a Precursor of the Solar Activity, Solar Phys., Vol. 260, pp. 465-477.

Tlatov, A. G. (2010a). The centenary variations in the solar corona shape in accordance with the observations during the minimal activity epoch, Astronomy and Astrophysics, Vol. 522, id.A27

Tlatov, A. G. (2010b). The Non-radial Propagation of Coronal Streamers within a Solar Cycle, ApJ, Vol. 714,pp. 805-809.

Vasil'eva, V. V. (1998). Recovery of large-scale synoptic maps of magnetic fields for the period 1880-1894, in Proceedings of the Conference "A New Cycle of Solar Activity: Observations and Theoretical Aspects" (in Russian), ed. A. V. Stepanov, (Pulkovo, St.Petersburg), 213-216.

Vsekhsvyatskiy, S. K., G. M. Nikolskiy, V. I. Ivanchuk, A. T. Nesmyanovich, et al. (1965). Solar Corona and Corpuscular Radiation in the Interplanetary Space, (Kiev, Naukova Dumka), 293.

Vsekhsvyatskiy, S. K., G. M. Nikolskiy, V. I. Ponamarev E.A. \& Cherednichenko V. I. (1955). On the corpuscular radiation from the sun, Astronomy Rep., Vol. 32, pp. 165-176

Waldmeier, M. (1976). Astron. Mitt. Eidgen. Sternw. Zurich, Vol. 351,pp. 13. 
Wang Y.-M. (1996). Nonradial Coronal Streamers, ApJ, Vol. 456, pp. L119-L121.

Wang, Y.-M., \& Sheeley, N. R., Jr. (1990). Solar wind speed and coronal flux-tube expansion, ApJ, Vol. 355, pp. 726-732

Zhao, X. P. \& Webb, D. F. (2003). Source regions and storm effectiveness of frontside full halo coronal mass ejections JGR, Vol. 108, pp. SSH 4-1. 


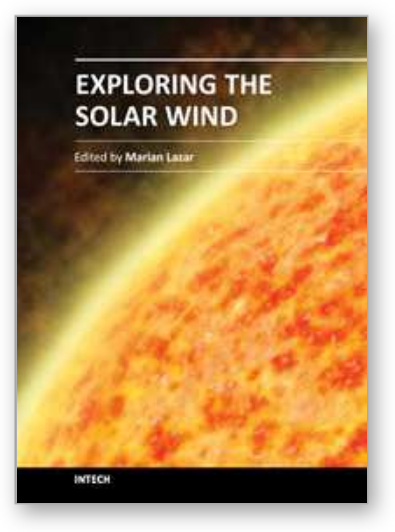

\author{
Exploring the Solar Wind \\ Edited by Dr. Marian Lazar
}

ISBN 978-953-51-0339-4

Hard cover, 462 pages

Publisher InTech

Published online 21, March, 2012

Published in print edition March, 2012

This book consists of a selection of original papers of the leading scientists in the fields of Space and Planetary Physics, Solar and Space Plasma Physics with important contributions to the theory, modeling and experimental techniques of the solar wind exploration. Its purpose is to provide the means for interested readers to become familiar with the current knowledge of the solar wind formation and elemental composition, the interplanetary dynamical evolution and acceleration of the charged plasma particles, and the guiding magnetic field that connects to the magnetospheric field lines and adjusts the effects of the solar wind on Earth. I am convinced that most of the research scientists actively working in these fields will find in this book many new and interesting ideas.

\title{
How to reference
}

In order to correctly reference this scholarly work, feel free to copy and paste the following:

A.G. Tlatov and B.P. Filippov (2012). Impact of the Large-Scale Solar Magnetic Field on the Solar Corona and Solar Wind, Exploring the Solar Wind, Dr. Marian Lazar (Ed.), ISBN: 978-953-51-0339-4, InTech, Available from: http://www.intechopen.com/books/exploring-the-solar-wind/impact-of-the-large-scale-solar-magneticfield-on-the-solar-corona-and-solar-wind

\section{INTECH}

open science | open minds

\section{InTech Europe}

University Campus STeP Ri

Slavka Krautzeka 83/A

51000 Rijeka, Croatia

Phone: +385 (51) 770447

Fax: +385 (51) 686166

www.intechopen.com
InTech China

Unit 405, Office Block, Hotel Equatorial Shanghai

No.65, Yan An Road (West), Shanghai, 200040, China

中国上海市延安西路65号上海国际贵都大饭店办公楼 405 单元

Phone: +86-21-62489820

Fax: $+86-21-62489821$ 
(C) 2012 The Author(s). Licensee IntechOpen. This is an open access article distributed under the terms of the Creative Commons Attribution 3.0 License, which permits unrestricted use, distribution, and reproduction in any medium, provided the original work is properly cited. 